\title{
On Current Conversion between Particle Rapidity and Pseudorapidity Distributions in High Energy Collisions
}

\author{
Fu-Hu Liu, Ya-Hui Chen, Ya-Qin Gao, and Er-Qin Wang \\ Institute of Theoretical Physics, Shanxi University, Taiyuan, Shanxi 030006, China \\ Correspondence should be addressed to Fu-Hu Liu; fuhuliu@163.com
}

Received 27 June 2013; Accepted 29 September 2013

Academic Editor: Sakina Fakhraddin

Copyright (C) $2013 \mathrm{Fu}-\mathrm{Hu}$ Liu et al. This is an open access article distributed under the Creative Commons Attribution License, which permits unrestricted use, distribution, and reproduction in any medium, provided the original work is properly cited.

In high energy collisions, one usually needs to give a conversion between the particle rapidity and pseudorapidity distributions. Currently, two equivalent conversion formulas are used in experimental and theoretical analyses. An investigation in the present work shows that the two conversions are incomplete. Then, we give a revision on the current conversion between the particle rapidity and pseudorapidity distributions.

\section{Introduction}

High energy collisions are an important research field in particle and nuclear physics. In the collisions, a lot of particles are produced, and the rapidity and/or pseudorapidity distributions can be obtained and studied [1-3]. Usually, one needs to do a conversion between the rapidity and pseudorapidity distributions in the case of only one of the two distributions being obtained. There are two equivalent conversion formulas used in the current literature [4-11]. Naturally, one thinks that the two conversions are perfect in investigations of the rapidity and pseudorapidity distributions.

However, our incidental find shows that the two conversions are incomplete. In obtaining the Jacobian in the current literature [4-11], a nongiven quantity, namely, transverse momentum, is erroneously used as a given one, which renders an incomplete conversion. In this paper, we will give a reanalysis on the Jacobian. A revised conversion between the rapidity and pseudorapidity distributions will be presented.

\section{General Definition}

We consider a system of high energy projectile-target collisions. The incident projectile direction is defined as $o z$ axis, and the reaction plane is defined as $x o z$ plane. Let $E, p, p_{L}$, $p_{T}, m_{0}$, and $\theta$ denote, respectively, the energy, momentum, longitudinal momentum, transverse momentum, rest mass, and emission angle of a concerned particle. According to general textbooks on particle physics $[12,13]$, the rapidity (which is in fact the longitudinal rapidity) is defined by

$$
y \equiv \frac{1}{2} \ln \left(\frac{E+p_{L}}{E-p_{L}}\right)
$$

where

$$
\begin{gathered}
E=\sqrt{p^{2}+m_{0}^{2}}, \\
p_{L}=p \cos \theta .
\end{gathered}
$$

In the case of $p \gg m_{0}$, we have

$y \approx \frac{1}{2} \ln \left(\frac{p+p_{L}}{p-p_{L}}\right)=\frac{1}{2} \ln \left(\frac{1+\cos \theta}{1-\cos \theta}\right)=-\ln \tan \left(\frac{\theta}{2}\right) \equiv \eta$,

where $\eta$ is the pseudorapidity.

Because the condition of $p \gg m_{0}$ is not always satisfied, the pseudorapidity distribution (density function) $f_{\eta}(\eta)=$ $(1 / N)(d N / d \eta)$ and the rapidity distribution (density function) $f_{y}(y)=(1 / N)(d N / d y)$ are not approximately equal to each other, where $d N$ denotes the particle number in the pseudorapidity or rapidity bin and $N$ denotes the total number of considered particles. 


\section{Current Conversion}

To give a conversion between $d N / d \eta$ and $d N / d y$ in the case of one of them being obtained, one has two equivalent methods which are currently used in the literature [4-11]. According to $[4,5,11]$, the first conversion relation between $d N / d \eta$ and $d N / d y$ can be given by

$$
\frac{d N}{d \eta}=\frac{d N}{d y} \frac{d y}{d \eta}=\frac{p}{E} \frac{d N}{d y}
$$

where

$$
\begin{aligned}
\frac{p}{E} & =\frac{\sqrt{E^{2}-m_{0}^{2}}}{E}=\sqrt{1-\frac{m_{0}^{2}}{E^{2}}} \\
& =\sqrt{1-\left(\frac{m_{0}}{\sqrt{p_{T}^{2}+m_{0}^{2} \cosh y}}\right)^{2}} .
\end{aligned}
$$

Then, the first conversion is given by [4-6]

$$
\frac{d N}{d \eta}=\frac{p}{E} \frac{d N}{d y}=\sqrt{1-\left(\frac{m_{0}}{\sqrt{p_{T}^{2}+m_{0}^{2}} \cosh y}\right)^{2}} \frac{d N}{d y}
$$

where $\sqrt{p_{T}^{2}+m_{0}^{2}} \equiv m_{T}$ is the transverse mass. We see that the first conversion is related to $p_{T}$.

The second conversion is given in $[5,7-11]$. We have

$$
\begin{aligned}
\frac{d N}{d \eta} & =\frac{\cosh \eta}{\sqrt{1+m_{0}^{2} p_{T}^{-2}+\sinh ^{2} \eta}} \frac{d N}{d y}=\frac{\cosh \eta}{\sqrt{m_{T}^{2} p_{T}^{-2}+\sinh ^{2} \eta}} \frac{d N}{d y} \\
& =\frac{\cosh \eta}{\sqrt{\cosh ^{2} \eta+m_{0}^{2} p_{T}^{-2}}} \frac{d N}{d y},
\end{aligned}
$$

which is also related to $p_{T}$. In [11], a similar conversion which uses $m^{2} P^{-2}$ instead of $m_{0}^{2} p_{T}^{-2}$ in (7) is given, where $m=$ $350 \mathrm{MeV}, P=0.13 \mathrm{GeV}+0.32 \mathrm{GeV}(\sqrt{s} / 1 \mathrm{TeV})^{0.115}$, and $\sqrt{s}$ denotes the center-of-mass energy. The conversion used in [11] is a mutation of the second conversion.

We now give the eduction of the current conversion. According to [12],

$$
\begin{aligned}
y & =\frac{1}{2} \ln \left(\frac{E+p_{L}}{E-p_{L}}\right)=\frac{1}{2} \ln \left(\frac{\sqrt{p^{2}+m_{0}^{2}}+p_{L}}{\sqrt{p^{2}+m_{0}^{2}}-p_{L}}\right) \\
& =\frac{1}{2} \ln \left(\frac{\sqrt{p_{T}^{2} \cosh ^{2} \eta+m_{0}^{2}}+p_{T} \sinh \eta}{\sqrt{p_{T}^{2} \cosh ^{2} \eta+m_{0}^{2}}-p_{T} \sinh \eta}\right) .
\end{aligned}
$$

In the case of $p_{T}$ being a given quantity, we have

$$
\begin{aligned}
\frac{d y}{d \eta}= & \frac{1}{2} \cdot \frac{\sqrt{p_{T}^{2} \cosh ^{2} \eta+m_{0}^{2}}-p_{T} \sinh \eta}{\sqrt{p_{T}^{2} \cosh ^{2} \eta+m_{0}^{2}}+p_{T} \sinh \eta} \\
& \cdot \frac{d}{d \eta}\left(\frac{\sqrt{p_{T}^{2} \cosh ^{2} \eta+m_{0}^{2}}+p_{T} \sinh \eta}{\sqrt{p_{T}^{2} \cosh ^{2} \eta+m_{0}^{2}}-p_{T} \sinh \eta}\right) \\
=\frac{1}{2} \cdot & {\left[\frac{1}{\sqrt{p_{T}^{2} \cosh ^{2} \eta+m_{0}^{2}}+p_{T} \sinh \eta}\right.} \\
& \cdot \frac{d}{d \eta\left(\sqrt{p_{T}^{2} \cosh ^{2} \eta+m_{0}^{2}}+p_{T} \sinh \eta\right)} \\
& -\frac{1}{\sqrt{p_{T}^{2} \cosh ^{2} \eta+m_{0}^{2}}-p_{T} \sinh \eta}
\end{aligned}
$$

$$
\left.\cdot \frac{d}{d \eta}\left(\sqrt{p_{T}^{2} \cosh ^{2} \eta+m_{0}^{2}}-p_{T} \sinh \eta\right)\right]
$$$$
=\frac{1}{2} \cdot\left[\frac{1}{\sqrt{p_{T}^{2} \cosh ^{2} \eta+m_{0}^{2}}+p_{T} \sinh \eta}\right.
$$

$$
\begin{aligned}
& \times\left(\frac{p_{T}^{2} \cosh \eta \sinh \eta}{\sqrt{p_{T}^{2} \cosh ^{2} \eta+m_{0}^{2}}}+p_{T} \cosh \eta\right) \\
& -\frac{1}{\sqrt{p_{T}^{2} \cosh ^{2} \eta+m_{0}^{2}}-p_{T} \sinh \eta} \\
& \left.\times\left(\frac{p_{T}^{2} \cosh \eta \sinh \eta}{\sqrt{p_{T}^{2} \cosh ^{2} \eta+m_{0}^{2}}}-p_{T} \cosh \eta\right)\right]
\end{aligned}
$$

$$
\begin{aligned}
&=\frac{1}{2} \cdot \frac{1}{p_{T}^{2}+m_{0}^{2}} \\
& \cdot\left[\left(\frac{p_{T}^{2} \cosh \eta \sinh \eta}{\sqrt{p_{T}^{2} \cosh ^{2} \eta+m_{0}^{2}}}+p_{T} \cosh \eta\right)\right. \\
& \quad \times\left(\sqrt{p_{T}^{2} \cosh ^{2} \eta+m_{0}^{2}}-p_{T} \sinh \eta\right) \\
&-\left(\frac{p_{T}^{2} \cosh ^{2} \sinh \eta}{\sqrt{p_{T}^{2} \cosh ^{2} \eta+m_{0}^{2}}}-p_{T} \cosh \eta\right) \\
& \quad \times\left(\sqrt{p_{T}^{2} \cosh ^{2} \eta+m_{0}^{2}}+p_{T} \sinh \eta\right)
\end{aligned}
$$




$$
\begin{aligned}
& =\frac{1}{2} \cdot \frac{1}{p_{T}^{2}+m_{0}^{2}} \cdot \frac{2\left(p_{T}^{2}+m_{0}^{2}\right) p_{T} \cosh \eta}{\sqrt{p_{T}^{2} \cosh ^{2} \eta+m_{0}^{2}}} \\
& =\frac{p_{T} \cosh \eta}{\sqrt{p_{T}^{2} \cosh ^{2} \eta+m_{0}^{2}}} \\
& =\frac{\cosh \eta}{\sqrt{\cosh ^{2} \eta+m_{0}^{2} p_{T}^{-2}}}=\frac{p}{E}=\beta,
\end{aligned}
$$

where $\beta$ denotes the velocity of the concerned particle. Then, we obtain the current conversion.

However, we would like to point out that the previous conversion is incomplete due to the fact that $p_{T}=p / \cosh \eta$ is also a function of $\eta$, which should be considered in doing the differential treatment. Instead, $p$ and $E$ can be regarded as given quantities.

\section{Revised Conversion}

In the differential treatment, we think that both the $p_{T}=$ $p / \cosh \eta$ and $p_{L}=p \tanh \eta$ are functions of $\eta$. Contrarily, $p$ and $E$ have the fixed values for a given particle. Then,

$$
\begin{aligned}
y= & \frac{1}{2} \ln \left(\frac{E+p_{L}}{E-p_{L}}\right)=\frac{1}{2} \ln \left(\frac{E+p \tanh \eta}{E-p \tanh \eta}\right) \\
= & \frac{1}{2} \ln \left(\frac{1+\beta \tanh \eta}{1-\beta \tanh \eta}\right) \equiv h(\eta), \\
\frac{d y}{d \eta}= & \frac{1}{2} \cdot \frac{1-\beta \tanh \eta}{1+\beta \tanh \eta} \cdot \frac{d}{d \eta}\left(\frac{1+\beta \tanh \eta}{1-\beta \tanh \eta}\right) \\
= & \frac{1}{2} \cdot \frac{1-\beta \tanh \eta}{1+\beta \tanh \eta} \\
& \cdot\left[\frac{1}{1-\beta \tanh \eta}+\frac{1+\beta \tanh \eta}{(1-\beta \tanh \eta)^{2}}\right] \cdot \beta \frac{d}{d \eta}(\tanh \eta) \\
= & \frac{1}{2} \cdot \frac{1-\beta \tanh \eta}{1+\beta \tanh ^{2}} \cdot \frac{2 \beta}{(1-\beta \tanh \eta)^{2}} \cdot \frac{1}{\cosh ^{2} \eta} \\
= & \frac{\beta}{1-\beta^{2} \tanh ^{2} \eta} \cdot \frac{1}{\cosh ^{2} \eta} \\
= & \frac{\beta}{\cosh ^{2} \eta-\beta^{2} \sinh ^{2} \eta}=\frac{\beta}{1+\left(1-\beta^{2}\right) \sinh ^{2} \eta} \\
= & \frac{1-\left(1-\beta^{2}\right) \cosh ^{2} y}{\beta} \cdot
\end{aligned}
$$

It is different from the first conversion which gives that $d y /$ $d \eta=\beta$. Correspondingly,

$$
\begin{aligned}
\eta & =\frac{1}{2} \ln \left(\frac{p+p_{L}}{p-p_{L}}\right)=\frac{1}{2} \ln \left(\frac{p+E \tanh y}{p-E \tanh y}\right) \\
& =\frac{1}{2} \ln \left(\frac{\beta+\tanh y}{\beta-\tanh y}\right) \equiv \varphi(y),
\end{aligned}
$$

$$
\begin{aligned}
\frac{d \eta}{d y}= & \frac{1}{2} \cdot \frac{\beta-\tanh y}{\beta+\tanh y} \cdot \frac{d}{d y}\left(\frac{\beta+\tanh y}{\beta-\tanh y}\right) \\
= & \frac{1}{2} \cdot \frac{\beta-\tanh y}{\beta+\tanh y} \cdot\left[\frac{1}{\beta-\tanh y}+\frac{\beta+\tanh y}{(\beta-\tanh y)^{2}}\right] \\
& \cdot \frac{d}{d y}(\tanh y) \\
= & \frac{1}{2} \cdot \frac{\beta-\tanh y}{\beta+\tanh y} \cdot \frac{2 \beta}{\left(\beta-\tanh ^{2}\right.} \\
& \cdot \frac{1}{\cosh ^{2} y}=\frac{\beta}{\beta^{2}-\tanh ^{2} y} \cdot \frac{1}{\cosh ^{2} y} \\
= & \frac{\beta}{\beta^{2} \cosh ^{2} y-\sinh ^{2} y} \\
= & \frac{\beta}{1-\left(1-\beta^{2}\right) \cosh ^{2} y}=\frac{1+\left(1-\beta^{2}\right) \sinh ^{2} \eta}{\beta} .
\end{aligned}
$$

The expressions after the last equal marks in (11) and (13) are obtained from the expressions before the last equal marks in (13) and (11), respectively. It is obvious that the eduction of the revised conversion is simpler than that of the current conversion.

To use (10)-(13), we have relations

$$
\begin{aligned}
f_{\eta}(\eta)|d \eta|= & f_{y}(y)|d y|=f_{y}[h(\eta)] \\
& \cdot\left|\frac{\beta}{1+\left(1-\beta^{2}\right) \sinh ^{2} \eta}\right| \cdot|d \eta|, \\
f_{y}(y)|d y|= & f_{\eta}(\eta)|d \eta|=f_{\eta}[\varphi(y)] \\
& \cdot\left|\frac{\beta}{1-\left(1-\beta^{2}\right) \cosh ^{2} y}\right| \cdot|d y| .
\end{aligned}
$$

Then, we have further

$$
\begin{aligned}
& f_{\eta}(\eta)=f_{y}[h(\eta)] \cdot\left|\frac{\beta}{1+\left(1-\beta^{2}\right) \sinh ^{2} \eta}\right|, \\
& f_{y}(y)=f_{\eta}[\varphi(y)] \cdot\left|\frac{\beta}{1-\left(1-\beta^{2}\right) \cosh ^{2} y}\right| .
\end{aligned}
$$

Equations (15) and (16) translate the rapidity distribution to pseudorapidity one and the pseudorapidity distribution to rapidity one, respectively.

In the previous discussions,

$$
\beta=\sqrt{1-\left(\frac{m_{0}}{\sqrt{p_{T}^{2}+m_{0}^{2}} \cosh y}\right)^{2}}=\frac{\cosh \eta}{\sqrt{\cosh ^{2} \eta+m_{0}^{2} p_{T}^{-2}}}
$$

which can be used in the conversion. Then, the conversion is related to $p_{T}$ and $m_{0}$. To do a conversion, we need to know $p_{T}$ and $m_{0}$ for each particle. 


\section{Conclusion and Discussion}

We have given a revision on the current conversion between the particle rapidity and pseudorapidity distributions. It is shown that, comparing to the current first conversion, the revised one $((15)$ or $(16))$ has an additional term (1 $\left.\beta^{2}\right) \sinh ^{2} \eta$ or $-\left(1-\beta^{2}\right) \cosh ^{2} y$ in the denominator. In central rapidity region, $\sinh \eta \approx 0$ and $\cosh y \approx 1$; then, (15) and (16) change to the current conversion. However, in forward rapidity region, the difference between the revised conversion and current one is obvious.

Our conclusion does not mean that the current conversion between the unit-density functions $d^{2} N / d p_{T} d \eta$ and $d^{2} N / d p_{T} d y$, that is,

$$
\frac{d^{2} N}{d p_{T} d \eta}=\sqrt{1-\left(\frac{m_{0}}{\sqrt{p_{T}^{2}+m_{0}^{2}} \cosh y}\right)^{2}} \frac{d^{2} N}{d p_{T} d y},
$$

is also erroneous or incomplete [12]. In fact, the conversion between the two unit-density functions is correct due to $p_{T}$ being a series of fixed values in (18). To use (18), we also need to know $p_{T}$ and $m_{0}$ for each particle.

Because the conversion between rapidity and pseudorapidity distributions is not simpler than a direct calculation based on the definitions of rapidity and pseudorapidity, we would rather use the direct calculation in modeling analysis. In fact, in the epoch of high energy collider, the dispersion between rapidity and pseudorapidity distributions is small [7]. This means that we would also like to not distinguish strictly rapidity and pseudorapidity distributions in general modeling analysis.

\section{Conflict of Interests}

The authors declare that there is no conflict of interests regarding the publication of this paper.

\section{Acknowledgments}

This work was supported by the National Natural Science Foundation of China under Grant no. 10975095, the China National Fundamental Fund of Personnel Training under Grant no. J1103210, the Open Research Subject of the Chinese Academy of Sciences Large-Scale Scientific Facility under Grant no. 2060205, and the Shanxi Scholarship Council of China.

\section{References}

[1] S. Chatrchyan, V. Khachatryan, A. M. Sirunyan et al., "Measurement of the pseudorapidity and centrality dependence of the transverse energy density in $\mathrm{Pb}-\mathrm{Pb}$ collisions at $\sqrt{s_{\mathrm{NN}}}=$ 2.76 TeV," Physical Review Letters, vol. 109, no. 15, Article ID 152303, 16 pages, 2012.

[2] I. Bautista, C. Pajares, J. G. Milhano, and J. D. de Deus, "Rapidity dependence of particle densities in $p p$ and $A A$ collisions," Physical Review C, vol. 86, no. 3, Article ID 034909, 5 pages, 2012.
[3] B. Żabiński, "Methods of multiplicity reconstruction in heavy ion collisions in the ATLAS experiment," Acta Physica Polonica $B$, vol. 42, no. 7, pp. 1729-1736, 2011.

[4] M. Biyajima, M. Ide, T. Mizoguchi, and N. Suzuki, "Scaling behavior of $\left(N_{\mathrm{ch}}\right)^{-1} d N_{\mathrm{ch}} / d \eta$ at $\sqrt{S_{\mathrm{NN}}}=130 \mathrm{GeV}$ by PHOBOS collaboration and its analyses in terms of stochasticapproach," http://arxiv.org/abs/hep-ph/0110305.

[5] M. Biyajima, M. Ide, T. Mizoguchi, and N. Suzuki, "Scaling behavior of $\left(N_{\mathrm{ch}}\right)^{-1} d N_{\mathrm{ch}} / d \eta$ at $\sqrt{S_{\mathrm{NN}}}=130 \mathrm{GeV}$ by the PHOBOS collaboration and its implication," Progress of Theoretical Physics, vol. 108, no. 3, pp. 559-569, 2002.

[6] P. A. Steinberg, "Global observables at RHIC," Nuclear Physics A, vol. 698, no. 1-4, pp. 314c-322c, 2002.

[7] G. Wolschin, "Pseudorapidity distributions of produced charged hadrons in pp collisions at RHIC and LHC energies," Europhysics Letters, vol. 95, no. 6, Article ID 61001, 6 pages, 2011.

[8] D. Kharzeev and E. Levin, "Manifestations of high density QCD in the first RHIC data," Physics Letters B, vol. 523, no. 1-2, pp. 7987, 2001.

[9] D. M. Röhrscheid and G. Wolschin, "Centrality dependence of charged-hadron pseudorapidity distributions in $\mathrm{PbPb}$ collisions at energies available at the CERN large hadron collider in the relativistic diffusion model," Physical Review C, vol. 86, no. 2, Article ID 024902, 7 pages, 2012.

[10] C. Merino, C. Pajares, and Y. M. Shabelski, "Production of secondaries in high-energy d+Au collisions," European Physical Journal C, vol. 59, no. 3, pp. 691-703, 2009.

[11] J. L. Albacete, A. Dumitru, H. Fujii, and Y. Nara, "CGC predictions for $\mathrm{p}+\mathrm{Pb}$ collisions at the LHC," Nuclear Physics A, vol. 897, pp. 1-27, 2013.

[12] C. Y. Wong, Introduction to High-Energy Heavy-Ion Collisions, World Scientific, Singapore, 1994.

[13] N. S. Zhang, Particle Physics, Science Press, Beijing, China, 1986. 

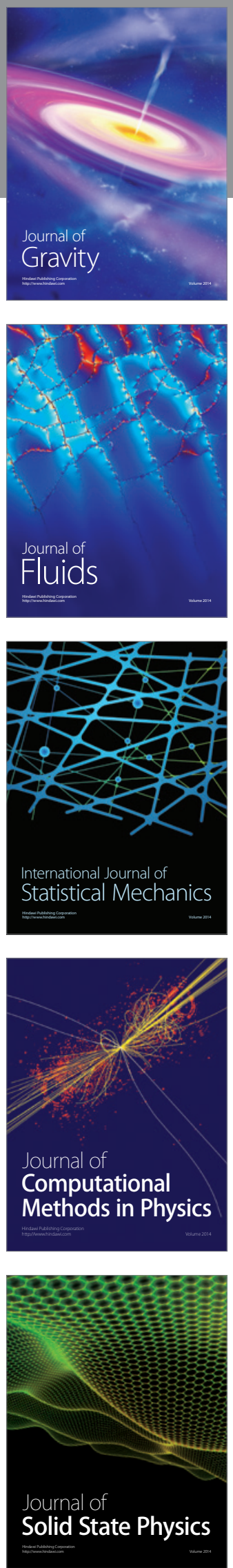

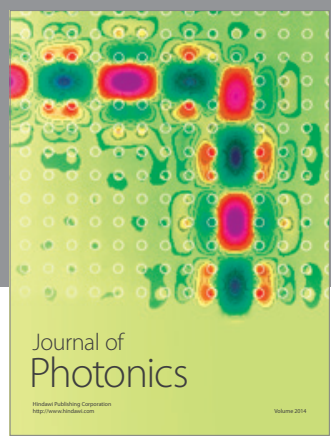

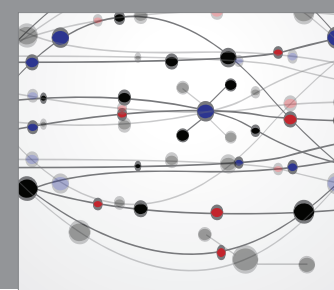

The Scientific World Journal

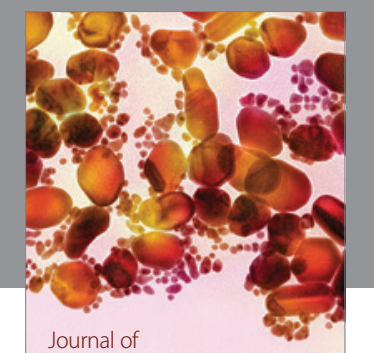

Soft Matter
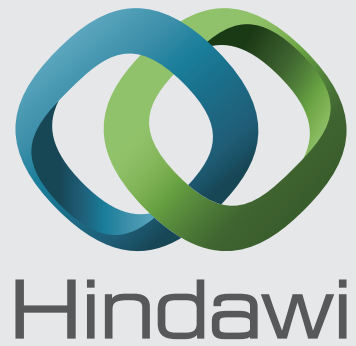

Submit your manuscripts at

http://www.hindawi.com
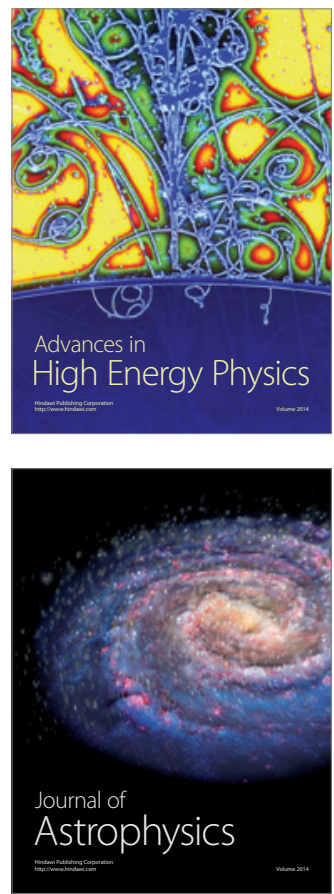
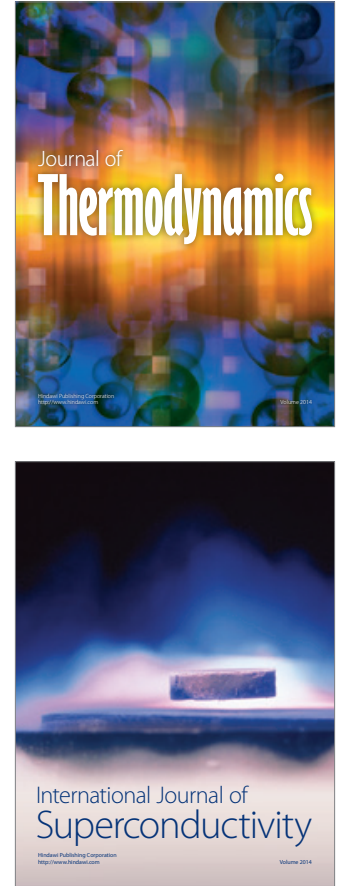
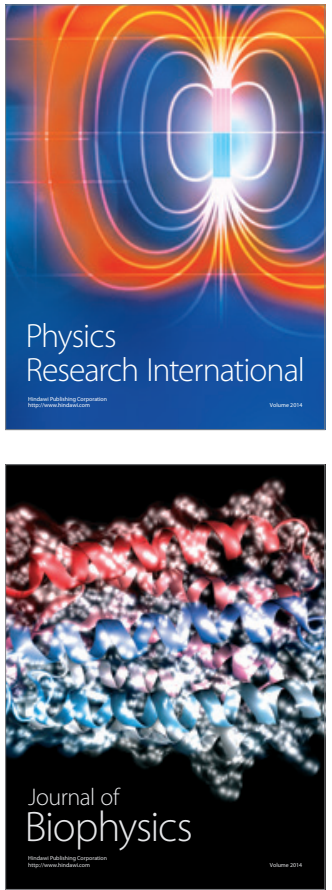
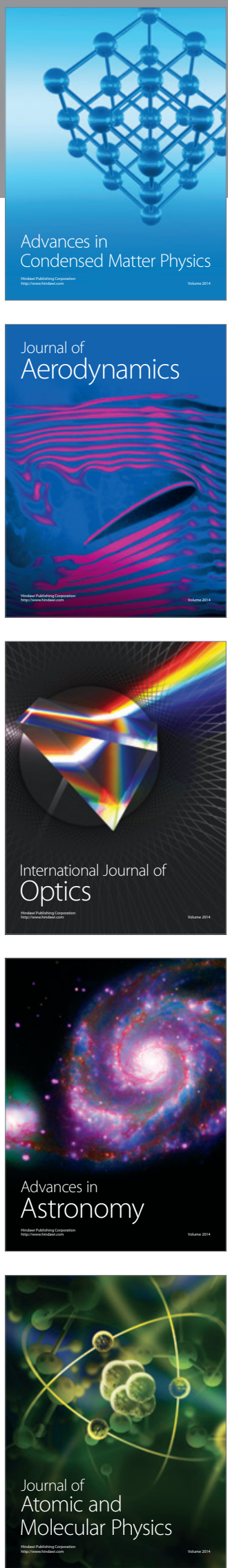